\title{
Fungal Infections of Oral Cavity: Diagnosis, Management, and Association with COVID-19
}

\author{
Arvind Babu Rajendra Santosh $^{1}$ (D) Keerthi Muddana ${ }^{2} \cdot$ Shobha Rani Bakki $^{3}$
}

Accepted: 22 March 2021 / Published online: 27 March 2021

(C) The Author(s), under exclusive licence to Springer Nature Switzerland AG 2021

\begin{abstract}
The frequency of fungal infections is increasing due to immunodeficiency viruses and immunosuppressive drugs. The most common fungal infection of the oral cavity is candidiasis. The existence of Candida can be a part of normal commensal; hence, the isolation of Candida in the absence of clinical symptoms should exclude candidiasis. The pathogenicity of Candida is witnessed as opportunistic when immune status is compromised. Oral fungal infections are uncommon, but when identified, these infections are associated with greater discomfort and are sometimes destruction of tissues. Cytology and tissue biopsy are helpful in confirming the clinical diagnosis. The management of oral fungal infections must strategically focus on signs, symptoms, and culture reports. This article reviews information on diagnosis and therapeutic management of aspergillosis, cryptococcosis, histoplasmosis, blastomycosis, mucormycosis, and geotrichosis.
\end{abstract}

Keywords Infection $\cdot$ Oral mycosis $\cdot$ Fungal $\cdot$ Candidiasis $\cdot$ Deep fungal infection

\section{Introduction}

The majority of oral fungal infections (oral mycosis) are resultant of opportunistic conditions. Host resistance impairment allows for the initiation and progression of pathogenic conditions through local colonization in the oral cavity. The frequency of oral mycosis has remarkably increased globally with the increased use of immunosuppressive drugs and immunodeficiency viral infections $[1,2]$. Oral mycological conditions range from superficial to deep fungal infections of the oral tissues. The most frequently diagnosed and reported oral fungal infections are the superficial type and candidiasis.

This article is part of theTopical Collection on Medicine

Arvind Babu Rajendra Santosh

arvindbabu2001@gmail.com

School of Dentistry, Faculty of Medical Sciences, The University of the West Indies, Mona, Kingston, Jamaica

2 Department of Oral and Maxillofacial Pathology, Tirumala Institute of Dental Sciences and Research Centre, Nizamabad, Telangana, India

3 Department of Oral Pathology, Meghna Institute of Dental Sciences, Nizamabad, Telangana, India
The predominance of oral candidiasis is being diagnosed probably due to the increasing number of individuals with immunocompromised conditions and dentists who are very experienced in the recognition and differentiation of oral candidiasis from other types of fungal infections [3, 4]. Whereas the clinical presentation of deep fungal infections is varied and not easily recognizable, this results in clinical diagnostic challenges [5]. Superficial fungal infections are usually associated with oral discomfort, pain, burning sensation, parageusia, and aversion to food $[4,6]$. Deep fungal infections are characterized by the dissemination of pathogens to the deeper areas of the tissue and are usually associated with aggressive clinical presentation such as ulceration and perforation into bony areas.

The diagnostic approach to oral mycotic conditions was focused on both clinical and cytological/histopathological examinations of the oral tissues. In most times, biopsy-based diagnosis helped in confirming clinically identified fungal conditions. In addition, microbial isolation, identification, culture, and antifungal susceptibility provided standard therapeutic care $[7,8]$, whereas the organism Candida may be cytologically/histological detected in an individual in the absence of a clinical oral condition, i.e., Candida can be seen in healthy individuals as a normal commensal. A microbiology report with a positive culture for Candida from an oral sample with no clinical presentation should eliminate the diagnosis of 
oral candidiasis, since Candida is a common commensal of the oral cavity. The clinical presentation of oral candidiasis is important in making the final diagnosis. Thus, clinical examination plays a major role in the diagnosis of superficial fungal infections such as candidiasis [9]. Oral candidiasis is usually a scrapable white lesion of the oral cavity. Hence, differential diagnosis of a scrapable white lesion must include oral candidiasis $[10,11]$.

The information presented in this paper was searched from available literature in PubMed and Google Scholar database. This paper discusses the diagnostic and therapeutic approaches to fungal infections of the oral cavity from the available research database and provides an update on oral fungal infections. Both common and uncommon oral mycoses such as candidiasis, aspergillosis, cryptococcosis, histoplasmosis, blastomycosis, mucormycosis, geotrichosis, and rhinosporidiosis are discussed. This review aims to provide oral fungal infections into categories, pathogenesis, clinical features, diagnosis, and management. The medications that are prescribed in the management of oral fungal infections are given in Table 1.

\section{Factors Influencing Epidemiology of Oral Mycoses and Sampling Methods}

The prevalence of fungal infections of the mucosa is increasing globally. Fungal infections usually develop because of aberrations in the immune system [4]. Oral mycosis is categorized as superficial and deep-seated infections. The most frequent type of oral mycosis is candidiasis $[12,13]$. The immunosuppressed state of an individual that includes human immunodeficiency virus infection, AIDS, malignancies, or diabetes is the most frequent cause of deep-seated infections [14]. The most frequent type of deep fungal infections is aspergillosis. Deep fungal infections which are observed in areas other than in oral tissue are indicators of systemic or disseminated infections. Various factors influence the epidemiology of opportunistic infections. Examples of this are organ transplant patients, corticosteroid-sparing regimens, exposure to azoles, better control of underlying systemic conditions, and prophylactic antibiotic regimens [15].

Recommended sampling methods for oral mycosis are the collection of whole saliva, rinsing sample, scraping over lesion/smear, moistened swab, imprint impression, blister fluid, or pus/inflammatory material from the active lesion. The moistened swab method is best suited for the oral fungal lesions that occur on the mucous membrane, lip, circumoral skin, and tongue. A prosthodontic impression, swab, or smear is the best sampling methods in denture stomatitis cases.

\section{Candidiasis}

The most frequent type of superficial fungal infection is candidiasis [16]. Sixty percentage of oral Candida were observed in a population of healthy, ambulatory, and nonimmunocompromised individuals in the oral and pharyngeal region [17].

Table 1 Medication management of oral fungal infections

\begin{tabular}{|c|c|c|}
\hline \multicolumn{2}{|l|}{ Medication } & \multirow{2}{*}{$\begin{array}{l}\text { Type of oral fungal infection } \\
\text { Oral candidiasis }\end{array}$} \\
\hline Oral suspension of nystatin & $100,000 \mathrm{IU} / \mathrm{ml}$ and $400,000-600,000 \mathrm{IU} / \mathrm{ml}$ as oral rinse & \\
\hline $\begin{array}{l}\text { Tablets clotrimazole and } \\
\text { fluconazole }\end{array}$ & $\begin{array}{l}\text { Tablet } 10 \mathrm{mg} \text { of clotrimazole and } 200 \mathrm{mg} \text { fluconazole administered on the first day, } \\
\text { followed by } 100-200 \mathrm{mg} \text { of fluconazole daily for } 7-14 \text { days }\end{array}$ & Oropharyngeal candidiasis \\
\hline Flucytosine & Flucytosine twice daily for $7-14$ days & Systemic candidiasis \\
\hline Oral suspension of flucystine & $10 \mathrm{mg} / \mathrm{ml}$ flucystine & Oropharyngeal candidiasis \\
\hline Amphotericin B & Local debridement with amphotericin B & Aspergillosis \\
\hline Fluconazole & Fluconazole $400 \mathrm{mg}$ for 6 months to 1 year & $\begin{array}{l}\text { Cryptococcosis (without } \\
\text { meningeal or pulmonary } \\
\text { symptoms) }\end{array}$ \\
\hline Itraconazole, amphotericin B & $\begin{array}{l}\text { Mild-to-moderate cases receive itraconazole after amphotericin B. } \\
\text { Moderate-to-severe cases are treated by amphotericin }\end{array}$ & Histoplasmosis \\
\hline $\begin{array}{l}\text { Itraconazole, amphotericin, } \\
\text { and azole }\end{array}$ & $\begin{array}{l}\text { Oral itraconazole is preferred in mild/moderate cases of pulmonary blastomycosis. } \\
\text { Amphotericin B and itraconazole are preferred in moderate/severe cases of } \\
\text { pulmonary blastomycosis with no CNS symptoms } \\
\text { Amphotericin and azole are preferred in pulmonary blastomycosis with CNS } \\
\text { symptoms }\end{array}$ & Blastomycosis \\
\hline $\begin{array}{l}\text { Amphotericin } \mathrm{B}, \\
\text { posaconazole }\end{array}$ & $\begin{array}{l}\text { Amphotericin B is a common drug of choice for mucormycosis. Posaconazole is } \\
\text { preferred in neutropenic patients }\end{array}$ & Mucormycosis \\
\hline Oral suspension of nystatin & $\begin{array}{l}\text { Treatment is similar to oral candidiasis. Amphotericin B syrup is also effective in } \\
\text { management }\end{array}$ & Geotrichosis \\
\hline
\end{tabular}




\section{Pathogenesis}

Specimens collected from an oral candidiasis lesion showed Candida albicans as the most frequent type of species. Other species in oral candidiasis lesions are Candida parapsilosis, Candida krusei, Candida stellatoidea, Candida tropicalis, Candida glabrata, Candida guilliermondii, and Candida dubliniensis. Although Candida is a common inhabitant of the oral mucosa, the growth of Candida is inhibited by other organisms in oral flora, and thus, lower levels of organisms are not enough to cause pathological alteration in the oral mucosal tissue. Systemic medications such as antibiotics, anti-cancer, immunosuppressive, and anti-cholinergic drugs are associated with oral candidiasis due to the alteration of the oral microbial flora that normally inhibits candidal growth [12]. Smoking, diabetes mellitus, nutritional disorders, endocrinopathies, immunosuppressive conditions, and malignancies are the factors that predispose oral candidiasis $[18,19]$. Decreased secretion of antimicrobial proteins are observed in individuals with hyposalivation. These include sialoperoxidase, lactoferrin, and lysozyme. Reduced levels of salivary antimicrobial proteins are related to decreased antifungal properties and may predispose the occurrence of oral candidiasis [20].

\section{Clinical Features}

Oral candidiasis is classified into pseudomembranous, erythematous, and hyperplastic forms. Angular cheilitis, denture stomatitis, and central papillary atrophy forms are Candidaassociated oral lesions. Candidiasis is also associated as a super-infection in leukoplakia, lichen planus, and lupus erythematosus. In generalized candidiasis, oral infections are a secondary site of infection [21]. Clinical appearance may vary in different forms of oral candidiasis. Pseudomembranous candidiasis is the most frequent type of oral candidiasis, which

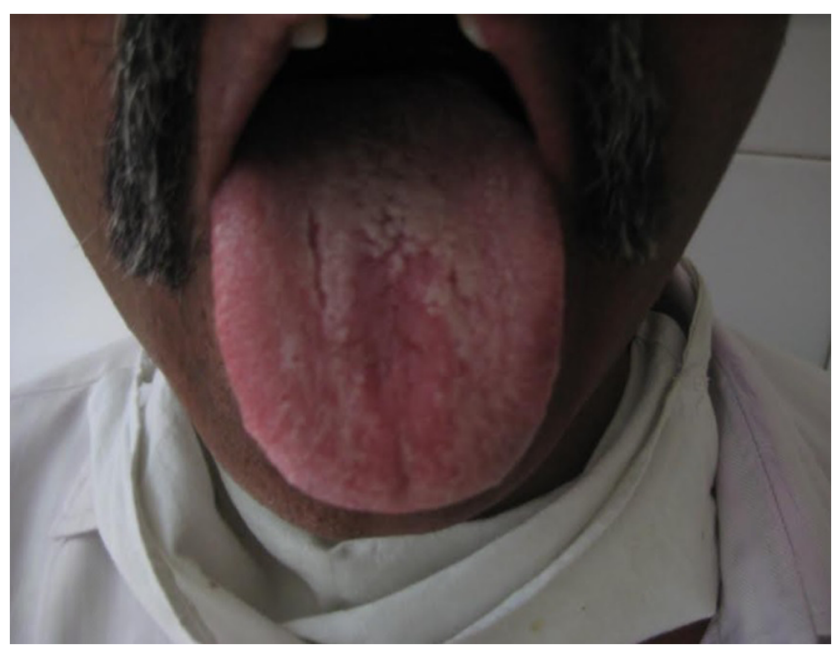

Fig. 1 Pseudomembranous Candidiasis presenting white scrapable plaques on the dorsum of tongue presents white or yellow scrapable plaques on the oral mucosa (Fig. 1). The white and/or yellow plaque on the oral mucosa is the yeast that predisposes keratinocyte desquamation. Pseudomembranous candidiasis can be either acute or chronic. The pseudomembranous form of oral candidiasis is most often times observed in patients with immune dysregulation or immunosuppressive medications [4]. The erythematous type can be either acute or chronic. The palate and dorsum of the tongue are the most common sites and are usually presented as atrophied areas of the oral mucosa which is characterized as erythematous patches. Erythematous candidiasis is commonly observed in individuals under corticosteroids or broadspectrum antibiotics. Chronic hyperplastic candidiasis presents as a white plaque on the commissural region of the oral cavity. The white plaque areas in chronic hyperplastic candidiasis cannot be readily scrapped off [21]. Denture-associated candidiasis is usually asymptomatic and may be presented with soreness at the denture-bearing area. The denture irregularities harbor the Candida and cause the bacterial microbes to grow. The growth of yeast in the denture is favored by the acidic and anaerobic environment in the tissue surface of the denture [22]. Clinically, denture-associated candidiasis is characterized by diffuse erythema and edema of the denturebearing area and is often associated with angular cheilitis. Central papillary atrophy is an uncommon type of candidiasis which is presented as a rhomboid-shaped atrophy or hypertrophy in the mid-dorsal tongue surface [23]. Angular cheilitis is a mixed form of infection with bacterial (streptococci and staphylococci) and fungal (Candida) organism involvement. Clinically, angular cheilitis is characterized by erythematous fissuring at the commissural areas.

\section{Diagnosis}

The clinical presentation of candidiasis is diagnostic, due to the classical white appearance [24]. The diagnostic methods for oral candidiasis include exfoliative cytology, imprint specimen for microbiology culture, potassium peroxide staining, oral swab specimen for culture analysis, salivary assays, and oral mucosal biopsy. Special staining procedure such as periodic acid-Schiff is helpful in definitive diagnosis [4]. Oral mucosal biopsy procedure is recommended in chronic hyperplastic candidiasis to differentiate it from leukoplakia and to evaluate the status of dysplasia [25]. Samaranayake et al. mentioned that chronic hyperplastic candidiasis may have a $15 \%$ chance of progression to epithelial dysplasia [26].

\section{Management}

Individuals with complaints of localized burning sensation in the oral cavity are sometimes managed with an empiric form of anti-candidiasis treatment. Due to the increased cost of laboratory investigations of candidiasis, most clinicians treat 
oral candidiasis with an empirical approach. The culture methods help in quantifying candidiasis organisms and differentiating between normal and increased levels of oral candidal infections [4]. Oral suspension of nystatin $(100,000 \mathrm{IU} / \mathrm{ml}$ and $400,000-600,000 \mathrm{IU} / \mathrm{ml}$ ) is available as oral rinse for oral candidiasis treatment, and patients should be guided to retain the suspension in the oral cavity prior to swallowing. The oral suspension of nystatin is stopped after $48 \mathrm{~h}$ after the disappearance of perioral symptoms. Central papillary atrophic candidiasis has the least tendency to resolve with antifungal treatment $[25,27]$. A few cases of endocrine-associated candidiasis remained refractory to nystatin treatment. Nystatin has shown favorable results with treating oral candidiasis. Oropharyngeal candidiasis is treated with $10 \mathrm{mg}$ of clotrimazole tablets. $200 \mathrm{mg}$ fluconazole tablets are administered on the first day of antifungal therapy, which is followed by 100 $200 \mathrm{mg}$ of fluconazole daily for 7-14 days. Fluconazole suspension is also available for oral rinse. Flucystine is used in the treatment of systemic candidiasis. $200 \mathrm{mg}$ of flucystine twice daily for 7-14 days and $10 \mathrm{mg} / \mathrm{ml}$ dose of flucystine oral rinse for candidiasis are recommended in the oropharyngeal manifestation of systemic candidiasis [4]. Disinfection of acrylic partial or complete dentures can be achieved by soaking in a $0.5 \%$ solution of sodium hypochlorite (i.e., household bleach should be diluted as 1:10 ratio with water) for $10 \mathrm{~min}$ [28]. In denture stomatitis or denture-related candidiasis cases, the patients should be guided to microwave disinfection of acrylic-based dentures, once per week. Microwave disinfection is as effective as the 14-day course of topical antifungal medication [29]. Minimal published reports are available on metal denture disinfection. The literature guides that metal-based dentures should be disinfected using chlorhexidine, hydrogen peroxide, and antifungal solutions [30].

\section{Aspergillosis}

Aspergillosis is the second most frequent oral mycosis and arises as a result of an opportunistic infection [9, 31, 32]. The most commonly identified species from cases of aspergillosis is Aspergillus fumigatus, followed by Aspergillus flavus, Aspergillus niger, and Aspergillus terreus. Aspergillosis affects individuals in immunosuppressive states and those with bone marrow transplantation in hematological malignancies [33]. Life-threatening complications can occur in immunocompromised patients with aspergillosis [4].

\section{Pathogenesis}

Aspergillosis organisms tend to invade vascular tissue, leading to thrombosis and infarction. In maxillary sinus aspergillosis, organisms can invade the oral tissue through the soft tissue lining of the maxillary sinus that progress down to the bone and cause palatal infarction and manifest in the oral mucosal tissue or disseminate into systemic organs.

Three forms of aspergillosis infections are invasive, noninvasive, and destructive non-invasive forms. The invasive type is characterized by the invasion of the fungal organism into tissues which lead to slow, progressive, and destructive or highly aggressive and lethal lesions, whereas the non-invasive forms of aspergillosis are classified as aspergilloma, fungal ball, mycetoma, and allergic Aspergillus sinusitis. Locally destructive lesions with no tissue invasion are observed in destructive non-invasive types of aspergillosis. The aspergillosis pathogen produces phospholipases, proteases, aflatoxin, gliotoxin, and hemolysis and phthioic acid that cause destruction and invasion [31].

Aspergillosis chiefly affects immunocompromised individuals, which may result in acute invasive aspergillosis of the lung tissue. Immunosuppressed individuals in hospitals are at a higher risk of nosocomial aspergillosis. This is due to the fungal species which have been identified in the hospital environment, especially where renovation takes place [34-36].

\section{Clinical Features}

Primary aspergillosis may be identified in the paranasal sinuses, larynx, eyes, ears, or oral cavity. Oral aspergillosis is typically characterized by black or yellow necrotic tissue on an ulcer base over the palate or in the posterior tongue. Toxins of aspergillosis hyphae help in penetrating the blood vessel wall and produce thrombosis that leads to infarction and necrosis. Aspergillosis organisms exhibit centrifugal linear growth and eventually develop into ball-shaped masses. The center of the mass contains calcium phosphate and is identified as foreign bodies on radiographic examination.

Inhalation of Aspergillus spores can lead to both upper and/ or lower respiratory infection resulting in pulmonary aspergillosis. Pulmonary aspergillosis may disseminate in the brain, bone, or endocardium. Invasion of the orbit and adjacent craniofacial areas can be seen in cases of chronic and/or aggressive invasive sino-nasal aspergillosis.

\section{Diagnosis}

Positive microbiological culture test results from a normally sterile area and histopathological examination are essential for final diagnosis. Host factor, clinical presentation and microbiological evidence make a diagnostic triad for aspergillosis. Two exceptions in the diagnosis of invasive aspergillosis are (1) histopathological examination shows the presence of hyphae in the absence of positive culture results and (2) satisfying the diagnostic criteria of aspergillosis with radiographic/ computer tomography findings and non-culture-based results in immunocompromised hosts with clinical presentations of 
aspergillosis. Microscopic examination of aspergillosis cases shows fungal hyphae that branch at $45^{\circ}$ angle. Conidiospores and fruiting bodies are also seen. It is also worth to note that hyphae of mucormycosis show non-septate hyphae that branch at $90^{\circ}$ angle $[31,37]$.

\section{Management}

Surgical management plays a significant role in treating invasive aspergillosis of sinus. Systemic medication management of aspergillosis is also essential in treating invasive aspergillosis. Published cases showed favorable responses with amphotericin B (AMB), voriconazole, itraconazole, and caspofungin. Local debridement with amphotericin B is employed as an adjunct treatment modality following surgical treatment. However, there are only few reports on the local debridement with amphotericin B. A randomized clinical trial (RCT) study reported that voriconazole is effective than deoxycholate amphotericin B (D-AMB) as a preliminary step in the management of invasive aspergillosis. Another RCT study compared liposomal amphotericin B (L-AMB), and their report revealed that liposomal therapy shall be considered as substitute preliminary management strategy in few patients [38].

\section{Cryptococcosis}

Cryptococcosis is another invasive fungal infection which affects the lungs as a primary target tissue and which may lead to the development of meningitis in later stages. Cryptococcus neoformans is a responsible pathogen for cryptococcosis, which presents substantial therapeutic challenges. Cryptococcus infections are commonly observed in immunocompromised and/or immunocompetent patients. Cryptococcus neoformans is the most frequent type of species isolated in immunocompromised individuals, whereas Cryptococcus gattii is similarly identified in immunocompetent individuals and healthy hosts.

\section{Pathogenesis}

The species Cryptococcus neoformans is commonly identified in dried pigeon feces and is sometimes isolated from fruits and soil. Cryptococcus neoformans can cause disease in animals. Animal-to-man and man-to-man modes of transmission have been reported. Reports suggest that a Cryptococcus infection in humans is acquired by the inhalation of infectious aerosols. The largest influence on the epidemiology of cryptococcosis was observed following to the evolution of an HIV infection. The issemination of Cryptococcus organisms is influenced by its polysaccharide capsule and secreted enzymes such as lactase, phospholipase B, and urease.

\section{Clinical Feature}

Cryptococcosis is a deep fungal infection characterized by invasive characteristics and often associated with morbidity and mortality. Cryptococcosis has a major role in causing oral infections and is an important opportunistic pathogen in both immunocompetent and immunocompromised individuals [39]. The most frequent sites for cutaneous cryptococcosis are the face, neck, and scalp [40]. Oral lesions are extremely rare but do occur in opportunistic condition. Oral presentation of cyrptococcosis includes mucosal surface ulceration, nodules, or granuloma formation. Oral ulcerations may have an induration which resembles carcinomatous tissue [41].

\section{Diagnosis}

The diagnosis of cryptococcosis requires histopathological documentation of the infection. The microscopic examination of cryptococcosis varies in patients. In immunocompetent hosts, biopsied tissue shows granulomatous areas with multinucleated giant cells that contain budding organisms in the form of intracytoplasmic Cryptococcus. Biopsied tissues from immunosuppressed patients show proliferation of both extraand intracellular Cryptococcus yeasts with few budding organisms. Neutrophils, lymphocytes, and macrophages are also observed in connective tissue stroma. Methenamine silver, mucicarmine, and periodic acid-Shiff (PAS) stains should be considered for definitive diagnosis. PAS-stained tissue will show bright magenta-colored fungal cytoplasm, and fungal capsules show positive stains using the mucicarmine staining method [42].

\section{Management}

The presentation of the infection occurs at single site but with no meningeal and/or pulmonary tissue involvement and no immunosuppressive risk factors; treatment of cryptococcosis is usually orally with fluconazole $400 \mathrm{mg}$ for 6 months to 1 year. Management of cryptococcosis infection in HIV-positive patients include induction, consolidation, and maintenance phases. Amphotericin and flucytosine are recommended in cryptococcal meningitis and extrapulmonary cryptococcal infections in the induction phase. Consolidation and maintenance therapy are initiated with oral fluconazole. In organ transplant patients, treating mild-to-moderate cryptococcosis of non-CNS disease is done with fluconazole. Treating moderately severe-to-severe non-CNS disease is with a combination of liposomal amphotericin and flucytosine [43]. 


\section{Histoplasmosis}

Histoplasmosis is a deep fungal infection that affects pulmonary tissue or mucocutaneous areas and is caused by histoplasmosis capsulatum. Histoplasmosis capsulatum is a dimorphic fungus with a yeast and mold form [44].

\section{Pathogenesis}

Histoplasma capsulatum is a pathogenic strain of the human tissue. When dimorphic pathogenic organisms are inhaled into the lungs, they change from the mold to the yeast form of fungus. Histoplasma capsulatum entry into the host tissue leads to subcellular localization, intracellular survival, and proliferation. Entry of the organism attracts neutrophils, macrophage, lymphocytes, and natural killer cells to the infected site. Macrophages assist in spreading the microorganism through the lymphatic and reticuloendothelial system of the host $[4,45]$.

\section{Clinical Features}

The mucocutaneous form of histoplasmosis may produce ulcerative, erosive lesions on the tongue, palate, and/or buccal mucosa. Oral lesions are frequently identified in cases with disseminated histoplasmosis. Oral manifestations are also observed in acute pulmonary histoplasmosis. Isolated cases of oral histoplasmosis are rarely documented. When oral lesions are present, they appear as solitary painful or painless ulcerations of the oral mucosa with a long-term duration of several weeks. Oral ulcerations are typically characterized by firm rolled borders that mimic a malignant neoplasm $[4,46]$.

\section{Diagnosis}

The diagnosis of histoplasmosis requires histopathological documentation of the infection. The microscopic examination of histoplasmosis is characterized by a chronic granulomatous condition, and the stroma shows multinucleated giant cells and macrophages. PAS and Grocott-Gomori methenamine special staining methods should be considered for definitive diagnosis. Individuals with pulmonary symptoms should be guided for chest radiographs [47].

\section{Management}

In mild-to-moderate histoplasmosis patients, itraconazole preferred azole therapy after receipt of AMB. Moderately severeor-severe histoplasmosis patients are treated with AMB. When patients receive AMB therapy, careful monitoring of electrolyte levels, blood cell count, and renal count should be done. Itraconazole should be avoided in patients receiving quinidine, pimozide, lovastatin, dofetilide, simvastatin, midazolam, or triazolam [48].

\section{Blastomycosis}

Blastomycosis is an endemic fungal infection reported in the Mississippi and Ohio River basins. Blastomyces dermatitidis is a spore-forming dimorphic fungi which is the responsible pathogen for blastomycosis. Blastomycosis is a deep fungal infection that most commonly affects the pulmonary, cutaneous, osseous, genitourinary, and central nervous system tissues [4].

\section{Pathogenesis}

Phagocytosis of alveolar macrophages, polymorpholeukocytes, and monocytes is responsible for inducing a natural resistance to Blastomyces dermatitidis infection. Phagocytosis action of alveolar macrophages helps in the inhibition of conidia to the formation of pathogenic yeast of Blastomyces. However, phagocytosis does not kill the ingested pathogenic strain due to the presence of a thick yeast capsule which leads to the proliferation of yeast forms in the alveoli region. The failure of natural resistance following the proliferation of yeast forms leads to non-caseating granuloma formation. Failure of the host response in pulmonary tissue leads to lympho-hematogenous dissemination taking place with the potential for foci of infection leading to multi-organ involvement [49].

\section{Clinical Feature}

Disseminated blastomycosis affects the oral cavity and is characterized by ulcerated lesions that are indurated and have an elevated margin [50]. The induration and elevated borders confound the lesion as a carcinomatous area [47].

\section{Diagnosis}

Clinical and radiographic details are not diagnostic in blastomycosis. Clinical manifestations of blastomycosis resemble other deep fungal infections and malignancy and often mimic tuberculosis. Growth of fungus or yeasts is the leading criteria for achieving a confirmatory or definitive diagnosis. The microscopic examination of blastomycosis is characterized by a chronic granulomatous condition, and the stroma often shows multinucleated giant cells and epithelioid histiocytes.

Demonstration of a double-refractive capsule of yeast cells is diagnostic. Broad-based budding versus non-budding yeast can be identified by Grocott-Gomori methenamine silver and PAS staining methods [49]. Immunoassays that detect the presence or absence of antigen or antibodies for blastomycosis are helpful in detecting the condition as an invasive fungal infection but are not specific for blastomycosis [51, 52]. 
Molecular methods such as nested polymerase chain reaction and nucleic acid amplification are useful in research studies but not for commercial or routine clinical detection [53].

\section{Management}

Management of blastomycosis is dependent on the anatomical site and severity of the infection. Acute and mild cases of blastomycosis may resolve spontaneously. However, the choice of antifungal medication type and duration may vary for individuals with a compromised immune system. In addition to the immune status of individual drug toxicity, drug-to-drug interaction and pregnancy will also influence selection of antifungal medication. Pulmonary blastomycosis (mild/moderate cases) or disseminated blastomycosis cases are managed by oral itraconazole [49]. Pulmonary blastomycosis (moderately severe-to-severe) or disseminated blastomycosis cases without involvement of the central nervous system (CNS) are managed by amphotericin B deoxycholate and itraconazole. Individuals with CNS involvement are managed by amphotericin $\mathrm{B}$, followed by azole therapy. Amphotericin B deoxycholate, lipid amphotericin B, and itraconazole are considered while managing blastomycosis cases in immunosuppressed individuals. Amphotericin $\mathrm{B}$ is preferred among pregnant individuals [54].

\section{Mucormycosis}

Mucormycosis is a deep fungal infection which occurs as acute and aggressive forms. Mucormycosis is caused by saprophytic fungi like Rhizopus, Mucor, Cunninghamella, Rhizomucor, Saksenaea, Apophysomyces, or Lichtheimia.

\section{Pathogenesis}

Mucormycosis primarily affects immunocompromised, bone marrow-transplanted, hematological malignancies, or poorly controlled diabetic individuals [33]. Rhizopus is the chief pathogenic mycotic organism in cases with rhinocerebral mucormycosis. Mucormycoses are identified on bread mold, soil, decaying vegetation, or animal manure. Cultures of swab obtained from the oral cavity, nasal cavity, throat, and stools of healthy persons may show mucormycoses. Fungal spore germinates to form hyphae in the host system upon entering tissues. Hyphae are responsible for the initiation of clinical symptoms, and persons with defective phagocytic function are at risk for developing an infection [55]. Impaired phagocytic functions increase the hyphae levels in the blood vessels, which results in ischemia, thrombosis, and finally infarction and tissue necrosis. In diabetic patients with ketoacidosis, the binding of iron to transferrin is inhibited and results in elevated iron levels, which promotes the growth of mucormycoses [56].

\section{Clinical Features}

Six well recognized clinical forms of mucormycosis are the pulmonary, cutaneous, gastrointestinal, rhinocerebral, central nervous system, and disseminated. Oral mucormycosis occurs usually in paranasal sinuses or nasal areas. Serious involvement of paranasal sinuses leads to palatal necrosis and/or ulceration $[57,58]$.

\section{Diagnosis}

Clinical presentation of mucormycosis usually provides an invasive picture of perforation into bony areas. Cases have been documented with oroantral communication or perforation extending to facial tissues. Confirmation of clinical diagnosis requires microscopic examination of the biopsied tissue $[59,60]$. The histopathological examination of tissue shows broad, non-septate type of hyphae with the pathognomonic nature of hyphae branching at right angles. The hyphae will be observed with a deeper connective tissue invasion. The special staining method, Grocott-Gomori methylamine silver stain will help in confirmation of the non-septate hyphae. Cases with bony perforations may show the presence of fungal organisms in marrow areas during histopathological examination. It is worth noting that cytological specimens collected from cases with histological diagnosis of mucormycosis may show the absence of organisms in fungal culture. A thorough clinical examination of the oral cavity in invasive lesions is recommended to achieve a clinical diagnosis, since not all the cases with mucormycosis will show the classical diagnostic interpretation in imaging studies like radiographs, computerized tomography (CT), magnetic resonance (MR), culture studies, or serological tests. Thus, a good clinical evaluation and histopathological examination remains the gold standard in diagnosis [61-63].

\section{Management}

Both medication and surgical management strategies are employed in mucormycosis cases. Amphotericin B (liposomal) is the most commonly used drug in the management of mucormycosis. Combination management of liposomal amphotericin B and posaconazole showed synergistic effects against fungal hyphae formation [64]. Neutropenic patients or individuals with graft-versus-host disease should be recommended for oral posaconazole medication as prophylactic management against mucormycosis, whereas mucormycosis cases in neutropenia or graft-versus-host disease patients should be managed by oral administration of fluconazole, while 
itraconazole and voriconazole are administered as prophylactic doses [65].

\section{Geotrichosis}

Geotrichosis is an uncommon opportunistic oral fungal infection caused by Geotrichum candidum. Clinical symptoms of geotrichosis resemble oral candidiasis and usually occur in immunocompromised individuals. Based on organ involvement, geotrichosis is classified into pulmonary, bronchial, intestinal, cutaneous, or mucosal (oral) types.

\section{Pathogenesis}

Geotrichum candidum is a low virulence type of the yeast form of a fungal organism. Geotrichum is one of the contaminant pathogens of water and milk. Geotrichum has been pathologically associated with the bronchi, lungs, and bowel tissues and less frequently in the oral cavity, skin, and nails. Geotrichum can be isolated from plants, vegetables, fruits, and soil. Pottier et al. mentioned Geotrichum candidum as a normal habitant of the oral mucosa [66]. However, a study stated that no molecular studies are available that isolated Geotrichum species as component of normal oral flora [67], whereas other studies have reported the Geotrichum species as a normal microbial inhabitant of the upper respiratory and gastrointestinal tract with a frequency of $30 \%$ in healthy individuals [68]. Geotrichosis (pulmonary or cutaneous types) is commonly reported among immunosuppressive disorders [69, 70]. Oral geotrichosis was reported in association with diabetes mellitus [67].

\section{Clinical Features}

Clinical appearance of geotrichosis mimics the pseudomembranous form of oral candidiasis [71]. Oral mucosal geotrichosis is characterized by a white, velvety, patch-like pseudomembranous appearance, palatal ulcerations, or villous hyperplastic areas. Twelve cases of oral geotrichosis association in non-insulin-dependent diabetes mellitus, Hodgkin's lymphoma, HIV infection, or acute lymphocytic leukemia have been reported [67]. Oral ulceration is a common manifestation of geotrichosis and should be considered in the differential diagnosis of ulcerative presentations in immunocompromised patients [67, 72]. However, confirmation of geotrichosis infection should be established after microscopic examination of a culture.

\section{Diagnosis}

Microscopic examination of a culture for the identification of the pathogenic organism is essential for the confirmation of a geotrichosis diagnosis. The microscopic examination of geotrichosis is characterized by small, rectangular-shaped spores with rounded edges. The pathologic tissue specimen shows a predominant acute inflammatory cellular infiltrate. Culture media available for geotrichosis includes Sabouraud dextrose agar (SDA), chloramphenicol, and CHROM agar media [67].

\section{Management}

Oral Geotrichosis responds well to treatment that is aimed at candidiasis [67]. In vitro susceptibility tests are effective in the management of oral geotrichosis. An in vitro susceptibility study mentioned that amphotericin B syrup is effective for managing oral geotrichosis [73]. Polacheck et al. reported variable sensitivity results of Geotrichum to flucytosine and sensitivity to amphotericin, miconazole, and ketoconazole [74]. Christakis et al. reported variable sensitivity of Geotrichum to flucytosine, itraconazole, and fluconazole [75]. The results of most studies suggest that amphotericin is a suitable antifungal agent and amphotericin syrup is effective at preventing systemic development in patients with oral geotrichosis [73].

\section{Rhinosporidiosis}

Rhinosporidiosis is a microbial infection characterized as a chronic granulomatous condition of the nasopharynx and oropharynx. Extra-nasal site involvement is reported in the eye, ear, trachea, parotid duct, skin, and genital mucosa. Rhinosporidiosis is caused by Rhinosporidium seeberi organism [76].

\section{Pathogenesis}

Rhinosporidiosis is a deep fungal infection that usually results from traumatic inoculation of the organism and most times is limited to the mucosal epithelium. Proliferation of pathogenic organisms is reflected in the association of tissue hyperplasia with a minimal immune response. The majority of rhinosporidiosis cases are sporadic. An ocular and skin rhinosporidiosis outbreak in swans was reported in the state of Florida in the USA [77].

\section{Clinical Features}

The clinical appearance of rhinosporidiosis is characterized by hyperplastic or proliferative tumor-like growths that are polypoid, lobulated (exophytic), and granular in nature [78]. Surface of oral lesion has yellow spots representing mature sporangia and erythematous areas due to increased vascularity. The growth is usually verrucous, and mucoid discharge may be seen. The lesion tends to readily bleed due to increased vascularity. Soft palatal lesions are common in oral 
rhinosporidiosis cases. Few reports have been reported with parotid duct involvement [76, 79]. Cases that show proliferative growth in parotid papillae regions should be suspected for oral rhinosporidiosis [76].

\section{Diagnosis}

The definitive diagnosis of rhinosporidiosis requires mucosal biopsy. Microscopic examination is characterized by large round-ovoid endospores consisting of sporangia [80]. The pathologic tissue shows increased vascularity and granulomatous changes with the presence of multinucleated giant cells. Cytodiagnoses on aspirates and on smears of secretion from the surface also have diagnostic value. Special staining such as PAS will help to differentiate endospores from epithelial cells. Endospores show magenta color in PAS staining, whereas epithelial cells do not show any specific staining characteristics. Detection of electron-dense bodies in endospores is required for definitive diagnosis of Rhinosporidium species [81].

\section{Management}

Surgery is the choice of treatment in rhinosporidiosis cases. Tissue growth observed in oral rhinosporidiosis is managed by surgical removal [80].

\section{Oral Fungal Infections Associated with COVID-19}

The pandemic severe acute respiratory syndrome coronavirus 2 (SARS-CoV-2) infection caused greatest health and social impact in the world since March 2020 [82]. A 67-year-old male patient who was tested positive for COVID-19 revealed his medical history of coronary disease and kidney transplant. Oral examination during hospital stay showed white plaque on the dorsum of the tongue. The patient was diagnosed with oral candidiasis [83]. Salehi et al. (2020) stated that COVID-19 patients at high risk included those have or have a history of respiratory distress syndrome, admitted in intensive care units, and used immunosuppressant drugs or corticosteroids are most likely to develop oral candidiasis [84]. Oral pharyngeal candidiasis was studied in hospitalized COVID-19 patients to identify the various Candida isolates. The study documented Candida albicans as the most frequent type of organism [85]. Another case study on a 47-year-old female patient who was tested positive for COVID-19 infection showed pseudomembranous candidiasis [86]. Interestingly, a published report of asymptomatic COVID-19 patient mentioned the presence of oral candidiasis [87]. Another published data revealed two patients with oral candidiasis in patients with no risk factors like immunosuppression or prolonged antibiotic use [88].

\section{Conclusion}

The clinical presentation of oral mycosis may vary depending on the pathogen. Oral mycosis may clinically present with a benign appearance with a change in color (white), swelling, or an invasive picture of an ulcerated swelling or ulcerative perforation with deep exposure of the bone. Oral mycosis must be suspected when the above-mentioned clinical presentations are found in immunocompromised patients or patients with immunosuppressive drugs. Critical evaluation of clinical data will assist dental surgeons in achieving a valid clinical diagnosis. Preliminary diagnosis of the oral mycosis can be achieved by salivary rinse or cytological methods. Hence, tissue sample collection for oral mycosis is minimally invasive.

Authors' Contribution All authors have read and approved the final manuscript. The principal author, ABRS made substantial contributions to the concepts, design, and intellectual content of the study and manuscript; has been involved in the preparation, editing, and review of the manuscript; and is the corresponding author. The co-author, KM, participated in manuscript writing concept, design, intellectual content of the study literature data acquisition, manuscript writing, and manuscript review. SRB participated in manuscript design and reviewed the intellectual content of the study literature and manuscript.

Data availability Not applicable.

\section{Declarations}

Ethical Approval The article is a narrative review from oral disease expert. Ethical approval is not applicable for this design of manuscript.

Informed Consent The article is a narrative review from oral disease expert. Informed consent is not applicable.

Conflict of Interest None.

\section{References}

1. Richardson M, Lass-Florl C. Changing epidemiology of systemic fungal infections. Clinical microbiology and infection. 2008;14(Suppl 4):5-24.

2. Nagy E. Changing epidemiology of systemic fungal infections and the possibilities of laboratory diagnostics. Acta microbiologica et immunologica Hungarica. 1999;46(2-3):227-31.

3. Singh A, Verma R, Murari A, Agrawal A. Oral candidiasis: an overview. Journal of oral and maxillofacial pathology : JOMFP. 2014;18(Suppl 1):S81-S5.

4. Samaranayake LP, Keung Leung W, Jin L. Oral mucosal fungal infections. Periodontology 2000. 2009;49(1):39-59. 
5. Hoepelman IM, Dupont B. Oral candidiasis: the clinical challenge of resistance and management. International Journal of Antimicrobial Agents. 1996;6(3):155-9.

6. Carmello JC, Alves F, G Basso F, de Souza Costa CA, Bagnato VS, Mima EGdO, et al. Treatment of oral candidiasis using Photodithazine $($-mediated photodynamic therapy in vivo. PloS one. 2016;11(6):e0156947-e.

7. Samaranayake YH, Samaranayake LP. Experimental oral candidiasis in animal models. Clinical microbiology reviews. 2001;14(2): 398-429.

8. Hoare A, Marsh PD, Diaz PI. Ecological therapeutic opportunities for oral diseases. microbiology spectrum. 2017;5(4):10.1128/ microbiolspec.BAD-0006-2016.

9. Krishnan PA. Fungal infections of the oral mucosa. Indian journal of dental research : official publication of Indian Society for Dental Research. 2012;23(5):650-9.

10. Coronado-Castellote L, Jimenez-Soriano Y. Clinical and microbiological diagnosis of oral candidiasis. Journal of clinical and experimental dentistry. 2013;5(5):e279-86.

11. Tsui C, Kong EF, Jabra-Rizk MA. Pathogenesis of Candida albicans biofilm. Pathogens and disease. 2016;74(4):ftw018-ftw.

12. Muzyka BC, Glick M. A review of oral fungal infections and appropriate therapy. The Journal of the American Dental Association. 1995; 126(1):63-72.

13. Santosh AB, Reddy BV. Oral mucosal infections: insights into specimen collection and medication management. Dental clinics of North America. 2017;61(2):283-304.

14. Farah CS, Lynch N, McCullough MJ. Oral fungal infections: an update for the general practitioner. Australian dental journal. 2010;55(Suppl 1):48-54.

15. Rajendra Santosh AB, Ogle OE, Williams D, Woodbine EF. Epidemiology of oral and maxillofacial infections. Dental clinics of North America. 2017;61(2):217-33.

16. Montelongo-Jauregui D, Lopez-Ribot J. Candida interactions with the oral bacterial microbiota. Journal of Fungi. 2018;4(4):122.

17. Fotos PG, Vincent SD, Hellstein JW. Oral candidosis: clinical, historical, and therapeutic features of 100 cases. Oral surgery, oral medicine, oral pathology. 1992;74(1):41-9.

18. Mead G. Management of oral mucositis associated with cancer chemotherapy. The Lancet. 2002;359(9309):815-6.

19. Ninane J, Group MS. A multicentre study of fluconazole versus oral polyenes in the prevention of fungal infection in children with hematological or oncological malignancies. European Journal of Clinical Microbiology and Infectious Diseases. 1994;13(4):330-7.

20. Akpan A, Morgan R. Oral candidiasis. Postgraduate medical journal. 2002;78(922):455-9.

21. Sitheeque M, Samaranayake L. Chronic hyperplastic candidosis/ candidiasis (candidal leukoplakia). Critical Reviews in Oral Biology \& Medicine. 2003;14(4):253-67.

22. Glass RT, Bullard JW, Hadley C, Mix E, Conrad R. Partial spectrum of microorganisms found in dentures and possible disease implications. The Journal of the American Osteopathic Association. 2001;101(2):65-6.

23. Redman RS. Prevalence of geographic tongue, fissured tongue, median rhomboid glossitis, and hairy tongue among 3,611 Minnesota schoolchildren. Oral Surgery, Oral Medicine, Oral Pathology. 1970;30(3):390-5.

24. Hellstein JW, Marek CL. Candidiasis: red and white manifestations in the oral cavity. Head and neck pathology. 2019;13(1):25-32.

25. Manfredi M, Polonelli L, Aguirre-Urizar J, Carrozzo M, McCullough M. Urban legends series: oral candidosis. Oral diseases. 2013;19(3):245-61.

26. Samaranayake LP, MacFarlane TW. Oral candidosis: Wright Publishing Company; 1990.
27. Rajendra Santosh AB, Boyd D, Laxminarayana KK. Clinical outline of oral diseases. Dental clinics of North America. 2020;64(1): $1-10$.

28. Ferreira MÁF, Pereira-Cenci T, de Vasconcelos LMR, RodriguesGarcia RCM, Cury AADB. Efficacy of denture cleansers on denture liners contaminated with Candida species. Clinical oral investigations. 2009;13(2):237-42.

29. Silva MM, de Oliveira Mima EG, Colombo AL, Sanitá PV, Jorge $\mathrm{JH}$, Massucato EMS, et al. Comparison of denture microwave disinfection and conventional antifungal therapy in the treatment of denture stomatitis: a randomized clinical study. Oral surgery, oral medicine, oral pathology and oral radiology. 2012;114(4):469-79.

30. Williams D, Lewis M. Pathogenesis and treatment of oral candidosis. Journal of oral microbiology. 2011;3(1):5771.

31. Deepa A, Nair BJ, Sivakumar T, Joseph AP. Uncommon opportunistic fungal infections of oral cavity: a review. Journal of oral and maxillofacial pathology : JOMFP. 2014;18(2):235-43.

32. Hartwick RW, Batsakis JG. Sinus aspergillosis and allergic fungal sinusitis. The Annals of otology, rhinology, and laryngology. 1991;100(5 Pt 1):427-30.

33. Vučićević Boras V, Jurlina M, Brailo V, Đurić Vuković K, Rončević P, Bašić Kinda S, et al. Oral mucormycosis and aspergillosis in the patient with acute leukemia. Acta stomatologica Croatica. 2019;53(3):274-7.

34. Steinbach WJ, Stevens DA. Review of newer antifungal and immunomodulatory strategies for invasive aspergillosis. Clinical Infectious Diseases. 2003;37(Supplement_3):S157-S87.

35. Staib F. Fungi in the home and hospital environment. Mycoses. 1996;39:26-9.

36. Shannon MT, Sclaroff A, Colm SJ. Invasive aspergillosis of the maxilla in an immunocompromised patient. Oral surgery, oral medicine, oral pathology. 1990;70(4):425-7.

37. Bathoorn E, Escobar Salazar N, Sepehrkhouy S, Meijer M, de Cock $\mathrm{H}$, Haas PJ. Involvement of the opportunistic pathogen Aspergillus tubingensis in osteomyelitis of the maxillary bone: a case report. BMC infectious diseases. 2013;13:59.

38. Walsh TJ, Anaissie EJ, Denning DW, Herbrecht R, Kontoyiannis DP, Marr KA, et al. Treatment of aspergillosis: clinical practice guidelines of the Infectious Diseases Society of America. Clinical infectious diseases. 2008;46(3):327-60.

39. Patel S, Navas M, Batt C, Jump RL. Oral cryptococcosis in a patient with chronic lymphocytic leukemia. International journal of infectious diseases. 2016;50:18-20.

40. Moore M. Cryptococcosis with cutaneous manifestations: four cases with a review of published reports1. Journal of Investigative Dermatology. 1957;28(2):159-82.

41. Cawley EP, Grekin RH, Curtis AC. Torulosis: a review of the cutaneous and adjoining mucous membrane manifestations. Journal of Investigative Dermatology. 1950;14(5):327-44.

42. Shibuya K, Hirata A, Omuta J, Sugamata M, Katori S, Saito N, et al. Granuloma and cryptococcosis. Journal of infection and chemotherapy. 2005;11(3):115-22.

43. Perfect JR, Dismukes WE, Dromer F, Goldman DL, Graybill JR, Hamill RJ, et al. Clinical practice guidelines for the management of cryptococcal disease: 2010 update by the Infectious Diseases Society of America. Clinical infectious diseases. 2010;50(3):291322.

44. Epifanio RN, Brannon RB, Muzyka BC. Disseminated histoplasmosis with oral manifestation. Special Care in Dentistry. 2007;27(6):236-9.

45. Woods JP, Heinecke EL, Luecke JW, Maldonado E, Ng JZ, Retallack DM, et al., editors. Pathogenesis of Histoplasma capsulatum. Semin Respir Infect; 2001.

46. Chroboczek T, Dufour J, Renaux A, Aznar C, Demar M, Couppie P, et al. Histoplasmosis: an oral malignancy-like clinical picture. Medical mycology case reports. 2018;19:45-8. 
47. Reddy BVR, Kuruba KK, Yalamanchili S, Mupparapu M. Granulomatous diseases affecting jaws. Dental Clinics. 2016;60(1):195-234.

48. Wheat LJ, Freifeld AG, Kleiman MB, Baddley JW, McKinsey DS, Loyd JE, et al. Clinical practice guidelines for the management of patients with histoplasmosis: 2007 update by the Infectious Diseases Society of America. Clinical Infectious Diseases. 2007;45(7):807-25.

49. Saccente M, Woods GL. Clinical and laboratory update on blastomycosis. Clinical Microbiology Reviews. 2010;23(2):367-81.

50. Albarillo FS, Varma GT, MacLeod SPR. Mandibular blastomycosis: a case report and review of the literature. Germs. 2018;8(4): 207-13.

51. Durkin M, Witt J, LeMonte A, Wheat B, Connolly P. Antigen assay with the potential to aid in diagnosis of blastomycosis. Journal of clinical microbiology. 2004;42(10):4873-5.

52. Turner S, Kaufman L, Jalbert M. Diagnostic assessment of an enzyme-linked immunosorbent assay for human and canine blastomycosis. Journal of clinical microbiology. 1986;23(2):294-7.

53. McCullough MJ, DiSalvo AF, Clemons KV, Park P, Stevens DA. Molecular epidemiology of Blastomyces dermatitidis. Clinical infectious diseases. 2000;30(2):328-35.

54. Chapman SW, Dismukes WE, Proia LA, Bradsher RW, Pappas PG, Threlkeld MG, et al. Clinical practice guidelines for the management of blastomycosis: 2008 update by the Infectious Diseases Society of America. Clinical infectious diseases. 2008;46(12): 1801-12.

55. Ibrahim AS, Spellberg B, Walsh TJ, Kontoyiannis DP. Pathogenesis of mucormycosis. Clinical Infectious Diseases. 2012;54(suppl_1):S16-22.

56. Hingad N, Kumar G, Deshmukh R. Oral mucormycosis causing necrotizing lesion in a diabetic patient: a case report. International Journal of Oral and Maxillofacial Pathology. 2012;3(3).

57. Muzyka BC, Epifanio RN. Update on oral fungal infections. Dental clinics of North America. 2013;57(4):561-81.

58. Brondfield S, Kaplan L, Dhaliwal G. Palatal mucormycosis. Journal of general internal medicine. 2018;33(10):1815

59. Reddy S, Kumar K, Sekhar C, Reddy R. Oral mucormycosis: need for early diagnosis!! Journal of Dr NTR University of Health Sciences. 2014;3(2):145-7.

60. Benites BM, Fonseca FP, Parahyba CJ, Arap SS, Novis YA, Fregnani ER. Extensive oral mucormycosis in a transplanted patient. The Journal of craniofacial surgery. 2017;28(1):e4-5.

61. Patel NR, Patel PA. A case report of mucormycosis with palatal ulcer: a rare clinical dilemma. Journal of the College of Physicians and Surgeons-Pakistan : JCPSP. 2018;28(9):721-3.

62. Skiada A, Lanternier F, Groll AH, Pagano L, Zimmerli S, Herbrecht $\mathrm{R}$, et al. Diagnosis and treatment of mucormycosis in patients with hematological malignancies: guidelines from the 3rd European Conference on Infections in Leukemia (ECIL 3). Haematologica. 2013;98(4):492-504.

63. Petrikkos G, Skiada A, Lortholary O, Roilides E, Walsh TJ, Kontoyiannis DP. Epidemiology and clinical manifestations of mucormycosis. Clinical Infectious Diseases. 2012;54(suppl_1): S23-S34.

64. Morace G, Borghi E. Invasive mold infections: virulence and pathogenesis of mucorales. International journal of microbiology. 2011;2012.

65. Cornely O, Arikan-Akdagli S, Dannaoui E, Groll A, Lagrou K, Chakrabarti A, et al. ESCMID $\uparrow$ and ECMM $\ddagger$ joint clinical guidelines for the diagnosis and management of mucormycosis 2013. Clinical Microbiology and Infection. 2014;20:5-26.

66. Pottier I, Gente S, Vernoux J-P, Guéguen M. Safety assessment of dairy microorganisms: Geotrichum candidum. International journal of food microbiology. 2008;126(3):327-32.
67. Bonifaz A, Vázquez-González D, Macías B, Paredes-Farrera F, Hernández MA, Araiza J, et al. Oral geotrichosis: report of 12 cases. Journal of oral science. 2010;52(3):477-83.

68. Heinic GS, Greenspan D, MacPhail LA, Greenspan JS. Oral Geotrichum candidum infection associated with HIV infection: a case report. Oral surgery, oral medicine, oral pathology. 1992;73(6):726-8.

69. Chang WW, Buerger L. Disseminated geotrichosis: case report. Archives of internal medicine. 1964;113(3):356-60.

70. Girmenia C, Pagano L, Martino B, D'Antonio D, Fanci R, Specchia $\mathrm{G}$, et al. Invasive infections caused by Trichosporon species and Geotrichum capitatum in patients with hematological malignancies: a retrospective multicenter study from Italy and review of the literature. Journal of clinical microbiology. 2005;43(4):1818-28.

71. Telles DR, Karki N, Marshall MW. Oral fungal infections: diagnosis and management. Dental clinics of North America. 2017;61(2): 319-49.

72. Listemann H, Schönrock-Nabulsi P, Kuse R, Meigel W. Geotrichosis of oral mucosa: Geotrichose der Mundschleimhaut. Mycoses. 1996;39(7-8):289-91.

73. Hattori H, Inoue C, Tomita Y, Kanbe T. A case of oral geotrichosis caused by Geotrichum capitatum in an old patient. Japanese journal of infectious diseases. 2007;60(5):300.

74. Polacheck I, Salkin I, Kitzes-Cohen R, Raz R. Endocarditis caused by Blastoschizomyces capitatus and taxonomic review of the genus. Journal of clinical microbiology. 1992;30(9):2318-22.

75. Christakis G, Perlorentzou S, Aslanidou M, Megalakaki A, Velegraki A. Fatal Blastoschizomyces capitatus sepsis in a neutropenic patient with acute myeloid leukemia: first documented case from Greece. Mycoses. 2005;48(3):216-20.

76. Panda S, Lenka S, Padhiary SK, Sahoo SR, Srivastava G. Rhinosporidiosis in the parotid duct: a rare case report. Journal of investigative and clinical dentistry. 2013;4(4):271-4.

77. Kennedy F, Buggage R, Ajello L. Rhinosporidiosis: a description of an unprecedented outbreak in captive swans (Cygnus spp.) and a proposal for revision of the ontogenic nomenclature of Rhinosporidium seeberi. Journal of medical and veterinary mycology. 1995;33(3):157-65.

78. Santosh ABR, Boyd D, Laxminarayana KK. Proposed clinicopathological classification for oral exophytic lesions. J Clin Diagn Res. 2015;9(9):ZE01-ZE8.

79. Mohapatra M, Banushree C. Two rare cases of rhinosporidiosis of parotid duct: case reports and review of literature. Annals of maxillofacial surgery. 2014;4(2):234 .

80. Arseculeratne S. Recent advances in rhinosporidiosis and Rhinosporidium seeberi. Indian journal of medical microbiology. 2002;20(3):119.

81. Beattie JM. Rhinosporidium kinealyi: a sporozoon of the nasal mucous membrane. The Journal of Pathology and Bacteriology. 1906;11(3):270-5.

82. RS AB. Dental management in coronavirus disease 2019 outbreak. Eur Dent Res Biomater J. 2020;1(1):1-3.

83. Amorim Dos Santos J, Normando AGC, Carvalho da Silva RL, De Paula RM, Cembranel AC, Santos-Silva AR, et al. Oral mucosal lesions in a COVID-19 patient: new signs or secondary manifestations? International journal of infectious diseases : IJID : official publication of the International Society for Infectious Diseases. 2020;97:326-8

84. Salehi M, Ahmadikia K, Badali H, Khodavaisy S. Opportunistic fungal infections in the epidemic area of COVID-19: a clinical and diagnostic perspective from Iran. Mycopathologia. 2020;185(4): 607-11.

85. Salehi M, Ahmadikia K, Mahmoudi S, Kalantari S, Jamalimoghadamsiahkali S, Izadi A, et al. Oropharyngeal candidiasis in hospitalised COVID-19 patients from Iran: species 
identification and antifungal susceptibility pattern. Mycoses. 2020;63(8):771-8.

86. Riad A, Gad A, Hockova B, Klugar M. Oral candidiasis in nonsevere COVID-19 patients: call for antibiotic stewardship. Oral surgery. 2020.

87. Corchuelo J, Ulloa FC. Oral manifestations in a patient with a history of asymptomatic COVID-19: case report. International journal of infectious diseases. 2020;100:154-7.
88. Baraboutis IG, Gargalianos P, Aggelonidou E, Adraktas A, Collaborators. Initial real-life experience from a designated COVID-19 centre in Athens, Greece: a proposed therapeutic algorithm. SN Compr Clin Med. 2020:1-5.

Publisher's Note Springer Nature remains neutral with regard to jurisdictional claims in published maps and institutional affiliations. 